\title{
A model for the $J-V$ characteristics of P3HT:PCBM solar cells
}

\author{
Pankaj Kumar, ${ }^{1,2, a)}$ S. C. Jain, ${ }^{1}$ Vikram Kumar, ${ }^{1}$ Suresh Chand, ${ }^{1}$ and R. P. Tandon ${ }^{2}$ \\ ${ }^{1}$ Centre for Organic Electronics, National Physical Laboratory, Dr. K. S. Krishnan Road, Pusa, \\ New Delhi 110012, India \\ ${ }^{2}$ Department of Physics and Astrophysics, University of Delhi, Delhi 110007, India
}

(Received 25 November 2008; accepted 7 April 2009; published online 22 May 2009)

\begin{abstract}
Current-voltage $(J-V)$ characteristics of an organic bulk heterojunction solar cell have been modeled and compared with the measured characteristics of solar cell based on the blend of poly(3-hexylethiophene) (P3HT) and phenyl $[6,6] \mathrm{C}_{61}$ butyric acid methyl ester (PCBM). In an undoped organic double Schottky junction diode, for $V<V_{\mathrm{bi}}$ the electric field remains constant and is given by $\left(V_{\mathrm{bi}}-V\right) / d$, where $V_{\mathrm{bi}}$ is the built-in voltage, $V$ is the applied voltage, and $d$ is sample thickness. We considered the effect of this constant electric field on the charge carrier transport and solved the drift and diffusion equations to model the $J$ - $V$ characteristics. For $V<V_{\mathrm{bi}}$ the current is found to be dominated by diffusion. A comparison of the theoretical results with the experimental data measured in dark and under different illumination intensities shows good agreement. (C) 2009 American Institute of Physics. [DOI: 10.1063/1.3129320]
\end{abstract}

\section{INTRODUCTION}

Organic semiconductors have proved to be quite revolutionary materials for electronic devices as they have found applications for thin, light weight, flexible, and low cost device fabrication. ${ }^{1}$ Intense research is being done on the next generation light emitting diodes and displays, solar cells, transistors, lasers, sensors, and other electronic devices based on organic semiconductors. ${ }^{2-5}$ The fast development has brought the organic light emitting diodes to the market place. Organic solar cells have been recognized as very important sources of energy and efforts are being made all over the world to improve their performance. The performance of organic solar cells has improved a lot in the last few years and the power conversion efficiency $(\eta)$ of $\sim 6.5 \%$ has successfully been achieved in a tandem structure of P3HT:PCBM solar cells. ${ }^{6}$ Long term stability is one of the major concerns for these devices and intense research is also being done to improve their stability. ${ }^{7-9}$ The protection from ambient may lead these devices to longer life and the organic solar cells having outdoor lifetime of several thousands of hours have successfully been fabricated. ${ }^{10}$ However further improvement in the performance needs the understanding of physics behind the operation of these devices. The illuminated current density in a conventional $(\mathrm{Si}) p-n$ junction solar cell is given by ${ }^{11}$

$$
J=J_{0}\left[\exp \left[\frac{q\left(V-J A R_{s}\right)}{n k T}\right]-1\right]+\frac{V-J A R_{s}}{R_{p} A}-J_{\mathrm{sc}},
$$

where $J_{0}$ is the reverse saturation current density, $q$ is elementary charge, $A$ is the active area of the device, $k$ is the Boltzmann's constant, $V$ is the applied voltage and $T$ is the absolute temperature, $R_{s}$ and $R_{p}$ are the series and parallel resistances, respectively, and $n$ is the ideality factor. $J_{\mathrm{sc}}$ is the photogenerated current density also known as short circuit

\footnotetext{
${ }^{a)}$ Author to whom correspondence should be addressed. Electronic mail: pankaj@mail.nplindia.ernet.in.
}

current density. The nature of dark and illuminated currents of an organic solar cell is different from that of one based on $\mathrm{Si}$. For example, no saturation is observed in the dark reverse current and the illuminated current becomes more than the dark current in forward bias. Therefore Eq. (1) is not valid for organic solar cells. The physics of characteristics of an organic solar cell is also not well understood therefore there has been considerable interest in the understanding of $J-V$ characteristics of organic bulk-heterojunction (BHJ) solar cells.

Generally the charge carrier mobility in the organics is very low and the current is governed by the space charge limited conduction for single Schottky diode. ${ }^{12-18}$ An organic solar cell consists of the organic active materials sandwiched between two metal electrodes forming two Schottky junctions and is quite similar to the metal-insulator-metal structure. If the two metal electrodes are of different work functions the Fermi level alignment results in the development of an internal electric field known as built-in electric field and the corresponding voltage developed at the two electrodes is known as the built-in voltage $\left(V_{\mathrm{bi}}\right)$. Depending upon the electrodes, $V_{\mathrm{bi}}$ can be as high as $\sim 1.3 \mathrm{~V}$ in organic solar cells. ${ }^{19}$ Therefore the effect of $V_{\mathrm{bi}}$ cannot be ignored in these devices. If there is no doping in the organic semiconductor and the metal contacts make large nonzero Schottky barriers with the organic, the electric field becomes constant throughout the active layer. ${ }^{19}$ Schilinsky et $a l^{20}{ }^{20}$ have shown the existence of constant electric field in the organic solar cells that remains constant even under intense illuminations. But the effect of this constant electric field on the dark current has not been taken into account. ${ }^{20}$ In this article we considered the effect of constant electric field and modeled the dark and illuminated $J-V$ characteristics for P3HT:PCBM BHJ solar cells. To elucidate the theoretical results the BHJ solar cells of P3HT:PCBM have been fabricated in indium tin oxide (ITO)/poly(ethylene dioxythiophene):poly styrene sulphonate (PEDOT:PSS)/P3HT:PCBM/Al configuration and measured their $J-V$ characteristics in dark and under different 


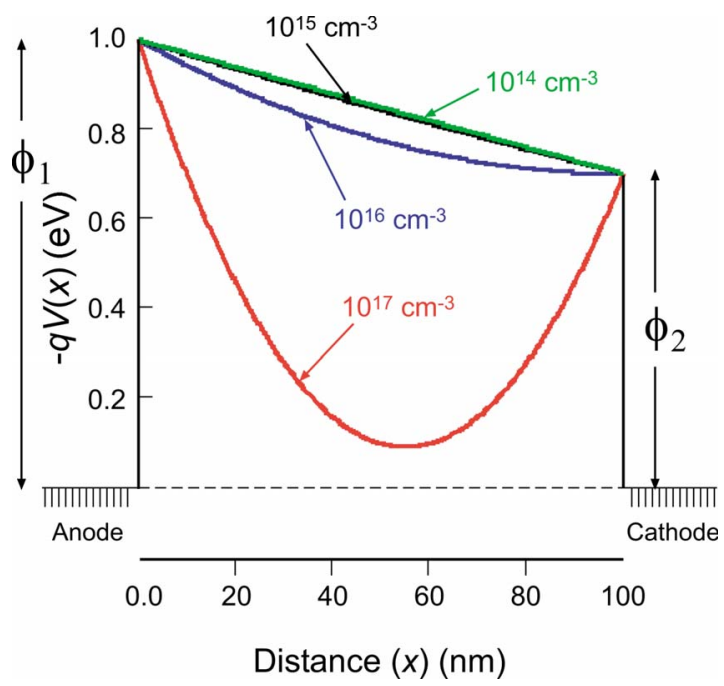

FIG. 1. (Color online) Variation in the band edge of the semiconductor with $x$ (in thermal equilibrium) for different $n$-type dopings (shown on the curves) of the semiconductor. The values of the other parameters are: $\phi_{1}$ $=1.0 \mathrm{eV}, \phi_{2}=0.7 \mathrm{eV}$, and $d=100 \mathrm{~nm}$. It is assumed that all the dopant atoms are active and ionized.

illumination intensities of tungsten-halogen lamp. A comparison of experimental data and the modeled results shows good agreement.

\section{THEORY}

Solving the Poisson's equation for the case of a doped semiconductor the conduction band edge is observed to vary with distance $x$ as

$-q V(x)=\frac{q}{\varepsilon \varepsilon_{0}} N_{d} \frac{x^{2}}{2}-\frac{1}{d}\left(\phi_{1}-\phi_{2}-q V+\frac{q}{\varepsilon \varepsilon_{0}} N_{d} \frac{d^{2}}{2}\right) x+\phi_{1}$,

where $N_{d}$ is the doping concentration of the active layer, $d$ is the thickness of the active layer, and $\phi_{1}$ and $\phi_{2}$ are the electron injection barriers from the anode and cathode, respectively. Figure 1 shows the plot of Eq. (2) as a function of $x$ $(x=0$ at the anode) in thermal equilibrium $(V=0 \mathrm{~V})$ for different doping concentrations $\left(N_{d}\right)$ of the active layer. It is seen from Fig. 1 that for large doping concentrations, the band edge and therefore the electric field $[F(x)=$ $-\partial V(x) / \partial x]$ depends strongly on the impurity concentration. As the impurity concentration decreases the electric field also decreases, first rapidly and then slowly. For a given set of parameters the band edges for $N_{d}=10^{14}$ and $10^{15} \mathrm{~cm}^{-3}$ differ very slightly. For the doping concentration of $10^{14} \mathrm{~cm}^{-3}$ or less the band edge becomes straight, and further reduction in doping concentration has practically no effect on it. The electric field now becomes constant and is solely determined by $\phi_{1}$ and $\phi_{2}$ and is given by $\left(\phi_{1}-\phi_{2}\right) / q d$ or $V_{\mathrm{bi}} / d$. Similar calculations were made for the case of an applied voltage $V$. The electric field now comes out to be $\left(V_{\mathrm{bi}}-V\right) / d$ provided $V$ is less than $V_{\mathrm{bi}}$. The results of Eq. (2) and Fig. 1 are strictly for inorganic semiconductors but with some suitable approximations can also be used for organic semiconductors as well. Mathematical calculations show that the electric field is constant in the organic semiconductor if the Schottky barri-
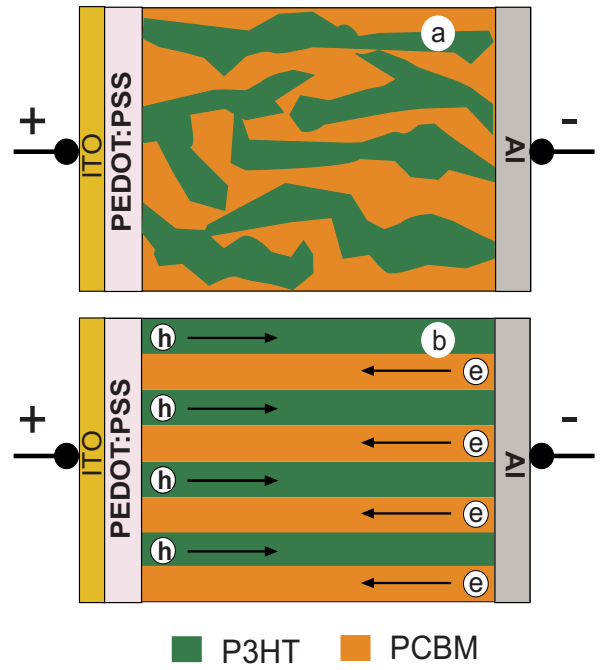

FIG. 2. (Color online) Schematic diagram of the conducting paths in the ITO/PEDOT:PSS/P3HT:PCBM/Al solar cell. (a) The interpenetrating P3HT and PCBM paths distributed in random zigzag way and (b) the distribution of $\mathrm{P} 3 \mathrm{HT}$ and PCBM used in the present modeling.

ers are large at both the contacts. ${ }^{19}$ If the Schottky contact is Ohmic the field becomes nonuniform and is large near the contact. Since in our case the Schottky barriers are not zero the electric field in the semiconductor may be assumed to be constant $\left(V_{\mathrm{bi}}-V\right) / d$ to a good approximation.

A schematic structure of the P3HT:PCBM blend sandwiched between ITO/PEDOT:PSS and Al electrodes is shown in Fig. 2. In the blend films P3HT and PCBM form the percolated paths, as shown in Fig. 2(a), and the charge carrier transport through these paths. For the purpose of modeling we replace the structure shown in Fig. 2(a) by that shown in Fig. 2(b). The holes are supposed to flow through P3HT channels and electrons through the PCBM channels. The electron and hole currents are modeled separately and the resultant terminal current is obtained by the combination of these two currents. Due to the fact that electrons and holes flow in different channels and are separated, the electrons and holes recombination is suppressed. Furthermore because of this spatial separation of the electrons and holes the interaction, which gives rise to ambipolar effect, is also reduced.

To model the $J-V$ characteristics of P3HT:PCBM solar cells we assume the P3HT:PCBM blend to be a single virtual semiconductor having electron and hole mobilities equal to electron mobility of PCBM and hole mobility of P3HT, respectively. The highest occupied molecular orbital (HOMO) and lowest unoccupied molecular orbital (LUMO) levels of the virtual semiconductor correspond to the HOMO of P3HT and LUMO of PCBM, respectively. The schematic energy level diagram of the solar cell is shown in Fig. 3. Figure 3(a) represents the energy levels of the materials used and the situation when the materials have not been brought into intimate contact. Figure 3(b) represents schematically the energy level diagram of the solar cell with virtual semiconductor before the intimate contact, and Fig. 3(c) shows the situation when the virtual semiconductor and contacting electrodes have been brought into intimate contact to formulate the cell. In the thermal equilibrium the electric field in 


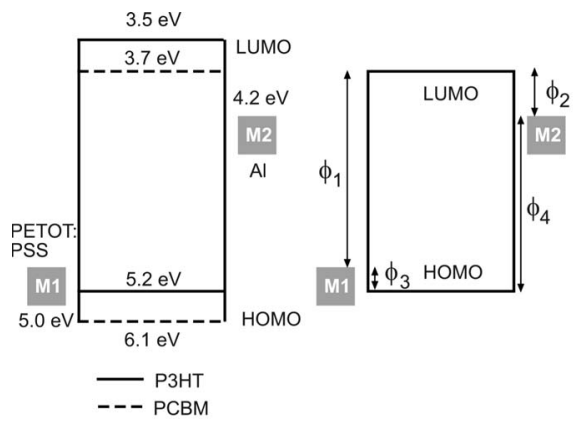

(a)

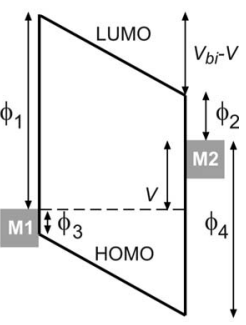

(c)

FIG. 3. Schematic energy level diagrams of the (a) PEDOT:PSS/ P3HT:PCBM/Al solar cell before the materials are brought into intimate contact; (b) virtual semiconductor (the blend of P3HT:PCBM is assumed to be a single virtual semiconductor) before it is brought into intimate contact with the electrodes; and (c) the virtual semiconductor is brought into intimate contact with the electrode materials.

the sample is equal to $V_{\mathrm{bi}} / d$, which reduces to $\left(V_{\mathrm{bi}}-V\right) / d$ when a forward bias voltage $V$ is applied to the sample.

\section{A. Dark current}

Let us first consider the case of electron current under forward bias (the negative terminal being connected to cathode), the continuity equation is given by

$$
J=q \mu n(x) F(x)+q D_{n} \frac{\partial n(x)}{\partial x},
$$

where $\mu$ is the charge carrier mobility, $n(x)$ is electron density, $F(x)$ is the electric field, and $D_{n}$ is the diffusion coefficient for electrons. Since $F(x)=-\partial V(x) / \partial x$, where $V(x)$ is the potential distribution in the semiconductor, Eq. (3) can be written as

$$
J=q D_{n}\left[-\frac{q}{k T} n(x) \frac{\partial V(x)}{\partial x}+\frac{\partial n(x)}{\partial x}\right] .
$$

Multiplying by the integrating factor $\exp [-q V(x) / k T]$ on both sides of Eq. (4) and integrating with respect to $x$ over the whole thickness of the active layer we get

$$
J=\frac{q D_{n}\left\{n(x) \exp \left[\frac{-q V(x)}{k T}\right]\right\}_{0}^{d}}{\int_{0}^{d} \exp \left[\frac{-q V(x)}{k T}\right] d x} .
$$

Using the boundary conditions ${ }^{11}$

$$
\begin{aligned}
& n(0)=N_{c} \exp \left(\frac{-\phi_{1}}{k T}\right), \\
& n(d)=N_{c} \exp \left(\frac{-\phi_{2}}{k T}\right),
\end{aligned}
$$

$$
\begin{aligned}
& q V(0)=-\phi_{1}, \\
& q V(d)=-\left(\phi_{2}+q V\right),
\end{aligned}
$$

where $N_{c}$ is the effective density of states, and $\phi_{1}$ and $\phi_{2}$ are the electron injection barriers at the anode and cathode, respectively. Eq. (5) gives

$$
J=\frac{q D_{n} N_{c}\left[\exp \left(\frac{q V}{k T}\right)-1\right]}{\int_{0}^{d} \exp \left[\frac{-q V(x)}{k T}\right] d x} .
$$

For the very low doping concentration of the semiconductor, Eq. (2) gives

$$
q V(x)=\left(\frac{\phi_{1}-\phi_{2}-q V}{d}\right) x-\phi_{1} .
$$

Assuming $\left(\phi_{1}-\phi_{2}\right)=q V_{\mathrm{bi}}$, Eq. (11) gives

$$
q V(x)=q\left(\frac{V_{\mathrm{bi}}-V}{d}\right) x-\phi_{1} .
$$

Using the value of $q V(x)$ from Eq. $\left(11^{\prime}\right)$, the denominator of Eq. (10) gives

$$
\begin{aligned}
\int_{0}^{d} \exp \left[\frac{-q V(x)}{k T}\right] d x= & \frac{d k T}{q\left(V_{\mathrm{bi}}-V\right)} \exp \left(\frac{\phi_{1}}{k T}\right) \\
& \times\left\{1-\exp \left[\frac{-q\left(V_{\mathrm{bi}}-V\right)}{k T}\right]\right\} .
\end{aligned}
$$

Now substituting this value in Eq. (10) we get

$$
J=\frac{q^{2} D_{n} N_{c}\left(V_{\mathrm{bi}}-V\right) \exp \left(\frac{-\phi_{1}}{k T}\right)\left[\exp \left(\frac{q V}{k T}\right)-1\right]}{d k T\left\{1-\exp \left[\frac{-q\left(V_{\mathrm{bi}}-V\right)}{k T}\right]\right\}} .
$$

Using Eqs. (4) and (13) the charge carrier distribution can now be given as 


$$
N(x)=\frac{N_{c} \exp \left(\frac{-\phi_{1}}{k T}\right)\left[\exp \left(\frac{q V}{k T}\right)-1\right]\left\{1-\exp \left[\frac{q\left(V_{\mathrm{bi}}-V\right)}{k T} \frac{x}{d}\right]\right\}}{\left\{\exp \left[-\frac{q\left(V_{\mathrm{bi}}-V\right)}{k T}\right]-1\right\}}+N_{c} \exp \left\{-\frac{\phi_{1}}{k T}+\left[\frac{q\left(V_{\mathrm{bi}}-V\right)}{k T} \frac{x}{d}\right]\right\} .
$$

Figure 4 shows the charge carrier distribution in thermal equilibrium and at $0.4 \mathrm{~V}$ forward bias. The values of the rest of the parameters are $T=295 \mathrm{~K}, \phi_{1}=0.88 \mathrm{eV}, V_{\mathrm{bi}}=0.58 \mathrm{~V}, d=100 \mathrm{~nm}$, and $N_{c}=1 \times 10^{19} \mathrm{~cm}^{-3}$. Equation (13) represents the $J-V$ characteristic of an ideal diode. For a practical diode Eq. (13) can now be modified to

$$
J=\frac{q^{2} D_{n} N_{c}\left(V_{\mathrm{bi}}-V+J A R_{s}\right) \exp \left(\frac{-\phi_{1}}{n k T}\right)\left\{\exp \left[\frac{q\left(V-J A R_{s}\right)}{n k T}\right]-1\right\}}{d n k T\left\{1-\exp \left[\frac{-q\left(V_{\mathrm{bi}}-V+J A R_{s}\right)}{n k T}\right]\right\}}+\frac{V-J A R_{s}}{R_{p} A} .
$$

The above equation takes correctly into account the effect of spreading of the carriers and increment in their concentration due to applied voltage.

Using Eq. (14) the diffusion and drift components of the current can now be calculated independently. Figure 5(a) shows the plot of diffusion and drift components of the electron current as a function of $x$. The currents have been calculated for $0.5 \mathrm{~V}$ and the values of rest of the parameters are given in the figure caption. Figure 5(b) shows the terminal current $\left(J_{\text {term }}\right)$ (which is the difference of the diffusion and drift components) at different voltages as a function of $x$. As expected the terminal current is observed to be independent of $x$ and increases with the applied voltage.

\section{B. Illuminated current}

Detailed computer simulation shows that under illumination also the electric field remains constant and is given by $\left(V_{\mathrm{bi}}-V\right) / d .^{20}$ The $J-V$ characteristics under illumination can now be given as

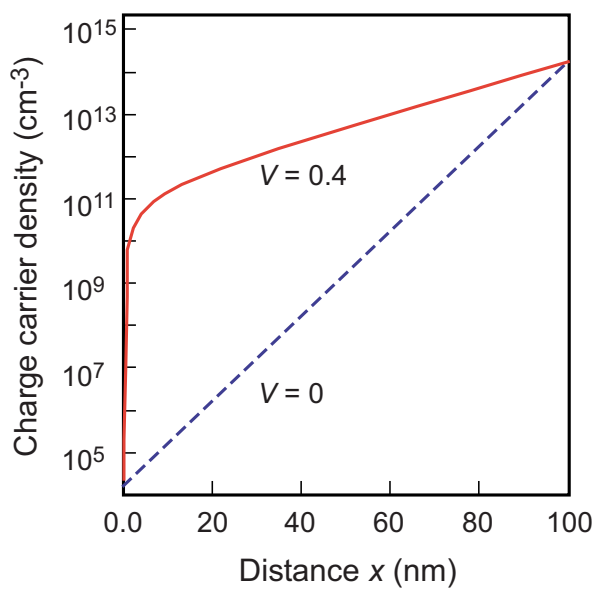

FIG. 4. (Color online) The injected electron profile in a semiconductor with cathode on the right hand side and anode on the left hand side. The dashed line is for thermal equilibrium and the solid line is for an applied voltage $=0.4 \mathrm{~V}$. The values of other parameters are: $T=295 \mathrm{~K}, \phi_{1}$ $=0.88 \mathrm{eV}, V_{\mathrm{bi}}=0.58 \mathrm{eV}, d=100 \mathrm{~nm}$, and $N_{c}=1 \times 10^{19} \mathrm{~cm}^{-3}$.

$$
\begin{aligned}
J= & \frac{q^{2} D_{n} N_{c}\left(V_{\mathrm{bi}}-V+J A R_{s}\right)}{\operatorname{dnkT}\left\{1-\exp \left[\frac{-q\left(V_{\mathrm{bi}}-V+J A R_{s}\right)}{n k T}\right]\right\}} \exp \left(\frac{-\phi_{1}}{n k T}\right) \\
& \times\left\{\exp \left[\frac{q\left(V-J A R_{s}\right)}{n k T}\right]-1\right\}+\frac{V-J A R_{s}}{R_{p} A}-J_{L}(V),
\end{aligned}
$$

where $J_{L}(V)$ is the photogenerated current. Series resistance is an important parameter and should always be considered therefore we have taken into account its effect on the internal electrical field. The applied voltage changes the internal electrical field which in tern will change the average carrier drift length $\left(D_{L}\right)$, given by

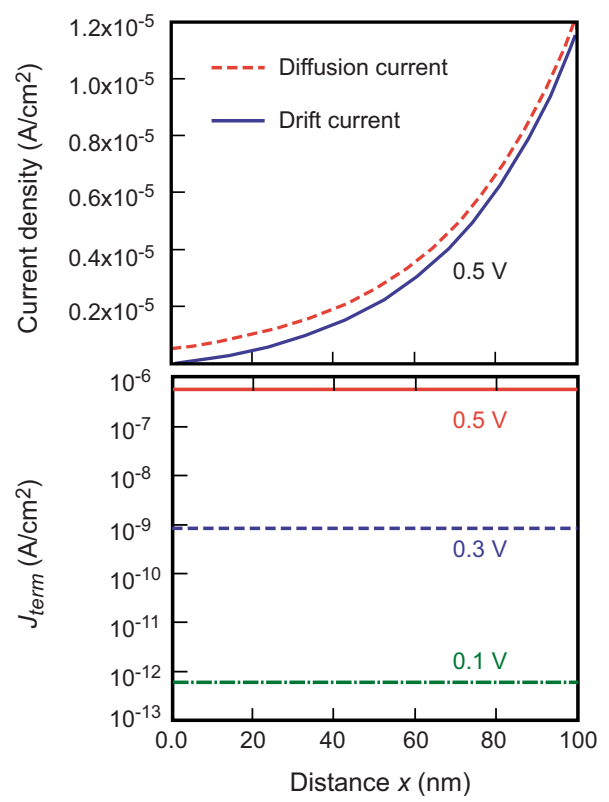

FIG. 5. (Color online) (a) Diffusion and drift currents at $300 \mathrm{~K}$ in the double Schottky barrier device at $0.5 \mathrm{~V}$. Diffusion current is larger than the drift current and the two currents flow in the opposite directions. The observed terminal current $\left(J_{\text {term }}\right)$ is the difference of these two currents. (b) The terminal currents $\left(J_{\text {term }}\right)$ are plotted at different applied voltages as a function of $x$. As expected the difference is independent of the values of $x$. The other values of the parameters are: $\phi_{1}=0.88 \mathrm{~V}, \phi_{2}=0.30 \mathrm{~V}, d=100 \mathrm{~nm}, N_{c}=1$ $\times 10^{19} \mathrm{~cm}^{-3}$, and $\mu=1 \times 10^{-4} \mathrm{~cm}^{2} \mathrm{~V}^{-1} \mathrm{~s}^{-1}$. 


$$
D_{L}(V)=\frac{\mu \tau\left(V_{\mathrm{bi}}-V+J A R_{S}\right)}{d},
$$

where $\tau$ is average lifetime of the carriers. The number of the carriers which can be extracted from the devices equals the total number of photogenerated carriers multiplied by the ratio of the average drift length to the thickness of the sample. Now the overall photogenerated current $\left(J_{L}\right)$ will now be given as ${ }^{20}$

$$
\begin{aligned}
& J_{L}(V)=\left|J_{\mathrm{sc}}\right| \quad \text { if } \mu \tau \frac{\left(-V+J A R_{s}+V_{\mathrm{bi}}\right)}{d}>d, \\
& J_{L}(V)=-\left|J_{\mathrm{sc}}\right| \quad \text { if } \mu \tau \frac{\left(V-J A R_{s}-V_{\mathrm{bi}}\right)}{d}>d, \\
& J_{L}(V)=\left|J_{\mathrm{sc}}\right| \mu \tau \frac{\left(-V+J A R_{S}+V_{\mathrm{bi}}\right)}{d^{2}} \text { else. }
\end{aligned}
$$

Similar expressions can be obtained for the hole current as well.

\section{EXPERIMENTAL DETAILS}

The BHJ solar cell of P3HT:PCBM has been fabricated in ITO/poly(ethylene dioxythiophene):poly(styrene sulphonate) (PEDOT:PSS)/P3HT:PCBM/Al configuration. All the organic materials were purchased from Sigma-Aldrich USA. The cell was fabricated on precleaned and prepatterned ITO coated glass substrate $(\sim 18 \Omega / \square)$. ITO substrate was cleaned using the standard cleaning procedure. ${ }^{21}$ Prior to any deposition the ITO substrate was exposed to oxygen plasma for five minutes. PEDOT:PSS ( 1.3 wt \% dispersion in $\mathrm{H}_{2} \mathrm{O}$ ) was spin coated on the ITO substrate at $1000 \mathrm{rpm}$ for $2 \mathrm{~min}$ and cured at $120^{\circ} \mathrm{C}$ for $1 \mathrm{~h}$ in a vacuum oven. P3HT and PCBM were individually dissolved in chlorobenzene and ultrasonicated for $30 \mathrm{~min}$ at $50{ }^{\circ} \mathrm{C}$. P3HT and PCBM content in their solutions was $20 \mathrm{mg} / \mathrm{ml}$. P3HT and PCBM solutions were mixed in 1:1 ratio and ultrasonicated for $30 \mathrm{~min}$. The P3HT:PCBM blend was spin coated on the PEDOT:PSS coated ITO substrate at $1000 \mathrm{rpm}$ for $2 \mathrm{~min}$ and cured at $140{ }^{\circ} \mathrm{C}$ for $15 \mathrm{~min}$ in a glove box. The final thicknesses of PEDOT:PSS and P3HT:PCBM blend film as measured from thickness profiler was found to be 45 and $110 \mathrm{~nm}$, respectively. Finally the sample was transferred to a vacuum chamber where $\mathrm{Al}$ electrodes $(500 \mathrm{~nm})$ were deposited via thermal evaporation in vacuum of about $2 \times 10^{-6}$ Torr through a shadow mask. The evaporation rate of $\mathrm{Al}$ was maintained $\sim 4-5 \AA / s$ by trial and error method. Thickness and rate of evaporation of $\mathrm{Al}$ electrodes were monitored using an oscillating quartz crystal thickness monitor. After fabrication the cell was transferred to a vacuum cryostat, where cell was mounted on a copper block. The copper block was connected to a stainless steel tube through which liquid nitrogen could be poured. To monitor the cell temperature a Pt 100 temperature sensor was mounted on copper block, very close to the cell. The $J-V$ characteristics of the cell were measured in dark and under illumination at $295 \mathrm{~K}$ using a Keithley 2400 Source Measure unit. The cell was illuminated with a tungsten-halogen lamp at different illumination intensities

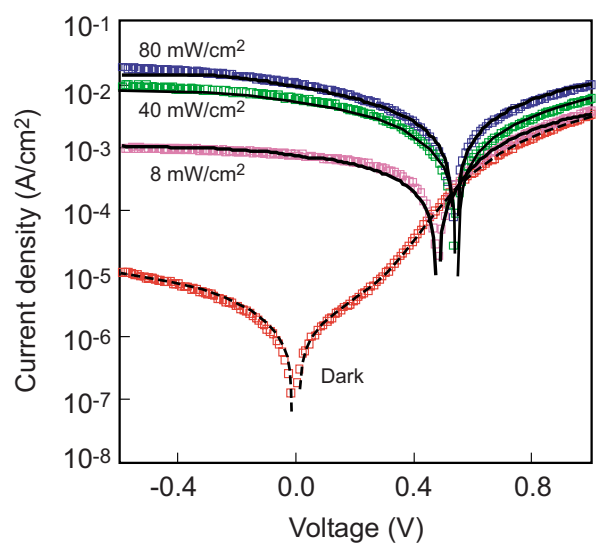

FIG. 6. (Color online) Calculated and experimental $J$ - $V$ characteristics of an ITO/PEDOT:PSS/P3HT:PCBM/Al solar cell in dark and under different illumination intensities. Symbols represent the experimental data. The solid curves represent the plot of Eq. (15) (for dark characteristic) and Eq. (16) (for illuminated characteristics). Values of the parameters used here are: $T$ $=295 \mathrm{~K}, \phi_{1}=1.3 \mathrm{~V}, \phi_{4}=0.9 \mathrm{~V}, \mu=1 \times 10^{-4} \mathrm{~cm}^{2} \mathrm{~V}^{-1} \mathrm{~s}^{-1}, \tau=2.5 \times 10^{-6} \mathrm{~s}$, $V_{\mathrm{bi}}=0.58 \mathrm{~V}, R_{s}=1 \Omega, A=0.1 \mathrm{~cm}^{2}, \varepsilon=3, n=1.85, d=150 \mathrm{~nm}, N_{c}=N_{v}$ $=1 \times 10^{19} \mathrm{~cm}^{-3}$, and $R_{p}=1.25 \times 10^{6}, 9.0 \times 10^{5}, 1.5 \times 10^{5}$, and 2.0 $\times 10^{4} \Omega$ for dark, 8,40 , and $80 \mathrm{~mW} / \mathrm{cm}^{2}$, respectively.

through a quartz window. The actual illumination intensity of the illuminating lamp was maintained at $80 \mathrm{~mW} / \mathrm{cm}^{2}$ and neutral density filters of different optical densities (ODs) $(0.0,0.3$, and 1.0$)$ were used to vary the illumination intensity. Note that at the time of light exposure the cell was getting heat. To avoid the heating effects on the cell the temperature of the cell was maintained at $295 \mathrm{~K}$ by the use of liquid nitrogen.

\section{COMPARISON OF THE MODEL WITH EXPERIMENTS}

The measured characteristics of the solar cell could be fitted with Eq. (1) but with the substantial variation in the values of the diode parameters under illumination. The variation in the values of the parameters under illumination cannot be justified therefore we compared the measured $J-V$ characteristics with those modeled and presented in this paper. Figure 6 shows the comparison of calculated terminal currents with the experimentally measured dark and illuminated currents of the P3HT:PCBM solar cell. Symbols represent the experimental dark and illuminated $J$ - $V$ characteristics of the cell at the respective illumination intensities and the dark curves represent the calculated characteristics. The values of the parameter are given in the caption of Fig. 6 . The device exhibited a maximum efficiency of $2.06 \%$ at $\sim 40 \mathrm{~mW} / \mathrm{cm}^{2}(\mathrm{OD}=0.3)$. Both the $J_{\mathrm{sc}}$ and $V_{\mathrm{oc}}$ are observed to increase with illumination intensity. $J_{\mathrm{sc}}$ shows a linear variation with the illumination intensity whereas increase in $V_{\text {oc }}$ decreases with light intensity, i.e., $V_{\text {oc }}$ tends to saturate. Qualitatively these trends are similar to those observed in $\mathrm{Si}$ photovoltaic devices. ${ }^{22}$ As discussed above the electrons and holes move in the separated percolated paths of PCBM and P3HT, respectively. Therefore the electron and hole currents have been calculated independently using, Eq. (15) and summed together to get the total terminal current of the cell in dark. Dashed curves in Fig. 6 represent the calculated 
terminal dark currents at different voltages. Similarly the calculated terminal currents of the cell under different illuminations have been obtained by the sum of illuminated currents of electrons and holes calculated using Eq. (16) for different illumination intensities. Solid curves represent the calculated terminal currents for respective illumination intensities. It has been observed that the modeled characteristics show good agreement with the measured characteristics for the same values of the diode parameters in dark and under different illumination intensities except in the parallel resistance $\left(R_{p}\right)$, which is observed to decrease with the increment in the illumination intensity. A reduction in the $R_{p}$ under illumination has also been observed by Schilinsky et al. ${ }^{20}$ The generation of charge carriers also lowers the resistance of the bulk via a photoinduced doping effect. This is done by adding a second photoconductive parallel resistor where the photoconductivity is proportional to the light intensity. In order to estimate the contribution of this effect to the parallel resistance $\left(R_{p}\right)$ the photoconductivity will have to be weighted by the probability of the charge carriers of one type to penetrate the barrier presented by the selective electrodes. Waldauf et al $^{23}$ called this weighing factor as the contact permeability (cp). The cp can be estimated by curve fitting to the $J-V$ characteristics of the illuminated solar cell under reverse bias. The behavior of the $V_{\text {oc }}$ under different light intensities leads to the determination of the otherwise difficult to evaluate life time $\tau$ of charge carriers, and the built-in voltage $V_{\mathrm{bi}}$ of the device. $V_{\mathrm{bi}}$ is observed to be not exactly equal to the difference of work functions of the electrodes but somewhat less and can be attributed to the loss in the vicinity of the PEDOT:PSS/P3HT contact. ${ }^{19}$ The comparison and the agreement of our model with experimental data obtained at various light intensities establish the validity of the model as a description for BHJ solar cells.

\section{CONCLUSIONS}

The $J-V$ characteristics of an organic solar cell have been modeled by solving the drift and diffusion equation for a constant electric field in the active layer. The drift and diffusion currents in the sample have been calculated separately, and for $V<V_{\mathrm{bi}}$ diffusion current is observed to be more than the drift current. As expected the terminal current is found to be independent of the distance in the sample and increases with the applied voltage. The modeled characteristics show good agreement with the experimental data measured in dark and at different illumination intensities. In actual BHJ solar cells the electrons and holes migrate by percolated paths in random zigzag paths. The model that we have presented here is therefore a simplified model which works well for actual BHJ photovoltaic devices.

\section{ACKNOWLEDGMENTS}

Authors would like to thank Dr. A. K. Gupta and Dr. S. S. Bawa for their help and support. Financial support by CSIR, India is acknowledged gratefully.

${ }^{1}$ S. C. Jain, M. Willander, and V. Kumar, Conducting Organic Materials and Devices (Academic, San Diego, 2007).

${ }^{2}$ E. Bundgaard and F. C. Krebs, Sol. Energy Mater. Sol. Cells 91, 954 (2007); H. Spanggaard and F. C. Krebs, ibid. 83, 125 (2004).

${ }^{3}$ S. Gunes, H. Neugebauer, and N. S. Sariciftci, Chem. Rev. (Washington, D.C.) 107, 1324 (2007); C. J. Brabec, N. S. Sariciftci, and J. C. Hummelen, Adv. Funct. Mater. 11, 15 (2001).

${ }^{4}$ P. E. Burrows, G. Gu, V. Bulovic, S. R. Forrest, and M. E. Thompson, IEEE Trans. Electron Devices 44, 1188 (1997); S. R. Forrest, Nature (London) 428, 911 (2004).

${ }^{5}$ H. Sirringhaus, Adv. Funct. Mater. 17, 2411 (2005).

${ }^{6}$ J. Y. Kim, K. Lee, N. E. Coates, D. Moses, T. Q. Nguyen, M. Dante, and A. J. Heeger, Science 317, 222 (2007).

${ }^{7}$ F. C. Krebs, Y. Thomann, R. Thomann, and J. W. Andreasen, Nanotechnology 19, 424013 (2008).

${ }^{8}$ M. Jørgensen, K. Norrman, and F. C. Krebs, Sol. Energy Mater. Sol. Cells 92, 686 (2008).

9 J. A. Hauch, P. Schilinsky, S. A. Choulis, R. Childers, M. Biele, and C. J. Brabec, Sol. Energy Mater. Sol. Cells 92, 727 (2008).

${ }^{10}$ C. Lungenschmied, G. Dennler, H. Neugebauer, S. N. Sariciftci, M. Glatthaar, T. Meyer, and A. Meyer, Sol. Energy Mater. Sol. Cells 91, 379 (2007).

${ }^{11}$ S. M. Sze, Physics of Semiconductor Devices (Wiley, New York, 1981).

${ }^{12}$ K. C. Kao and W. Hwang, Electrical Transport in Solids (Pergamon, New York, 1981).

${ }^{13}$ M. A. Lampert and P. Mark, Current Injection in Solids (Academic, New York, 1970).

${ }^{14}$ S. C. Jain, W. Geens, A. Mehra, V. Kumar, T. Aernouts, J. Poortmans, and R. Mertens, J. Appl. Phys. 89, 3804 (2001).

${ }^{15}$ P. Kumar, S. C. Jain, A. Misra, M. N. Kamalasanan, and V. Kumar, J. Appl. Phys. 100, 114506 (2006); P. Kumar, A. Misra, M. N. Kamalasanan, S. C. Jain, and V. Kumar, J. Phys. D 40, 561 (2007); A. Jain, P. Kumar, S. C. Jain, V. Kumar, and R. Kaur, J. Appl. Phys. 102, 094505 (2007).

${ }^{16}$ A. J. Campbell, M. S. Weaver, D. G. Lidzey, and D. D. C. Bradly, J. Appl. Phys. 84, 6737 (1998).

${ }^{17}$ T. Van-Woudenbergh, P. W. M. Blom, M. C. J. M. Vissenberg, and J. N. Huiberts, Appl. Phys. Lett. 79, 1697 (2001).

${ }^{18}$ N. F. Mott and R. W. Gurney, Electronic Processes in Ionic Crystals (Oxford University Press, New York, 1940).

${ }^{19}$ V. D. Mihailetchi, P. W. M. Blom, J. C. Hummelen, and M. T. Rispens, J. Appl. Phys. 94, 6849 (2003)

${ }^{20}$ P. Schilinsky, C. Waldauf, J. Hauch, and C. J. Brabec, J. Appl. Phys. 95, 2816 (2004).

${ }^{21}$ A. Misra, P. Kumar, S. K. Dhawan, M. N. Kamalasanan, and S. Chandra, Indian J. Pure Appl. Phys. 43, 522 (2005).

${ }^{22}$ S. C. Jain, E. L. Heasell, and D. J. Roulston, Prog. Quantum Electron. 11, 105 (1987)

${ }^{23}$ C. Waldauf, P. Schilinsky, J. Hauch, and C. J. Brabec, Thin Solid Films 451-452, 503 (2004) 